\title{
Turbulent forced convection heat transfer in triangular cross sectioned helically coiled tube
}

\author{
Mohamed Elashmawy 1, 2, Lioua Kolsi 1,3,* \\ ${ }^{1}$ College of Engineering, Department of Mechanical Engineering, Haïl University, Haïl City, Saudi Arabia \\ 2Department of Engineering Science, Faculty of Petroleum and Mining Engineering, Suez University, Suez, Egypt \\ 3Unité de recherche de Métrologie et des Systèmes Energétiques, Ecole Nationale d'Ingénieurs, 5000 Monastir, University of \\ Monastir, Tunisia
}

\section{A RTICLE INFO}

\section{Article history:}

Received 11 May 2016

Received in revised form

7 July 2016

Accepted 8 July 2016

\section{Keywords:}

Helically coiled tube

Turbulent

Triangular cross section

\begin{abstract}
A B S T R A C T
A three-dimensional turbulent forced convective heat transfer and flow characteristic, in a helical triangular cross sectioned coiled tube is simulated using the $\mathrm{k}-\varepsilon$ standard turbulence model and compared to results corresponding to circular cross sectioned helical tube. A finite element method is employed to solve the governing equations. The effects of Reynolds number, on Nusselt number, velocity and temperature profiles are discussed. The numerical computations show the developments and distributions of heat transfer and flow fields in the tube. These profiles are altered by the effect of curvature inducing the centrifugal forces.
\end{abstract}

(C) 2016 The Authors. Published by IASE. This is an open access article under the CC BY-NC-ND license (http://creativecommons.org/licenses/by-nc-nd/4.0/).

\section{Introduction}

Optimization of forced convection heat transfer from helical coiled tubes has a great importance in many fundamental and engineering applications especially in heat exchangers and storage tanks technologies.

An important number of theoretical, numerical and experimental studies investigated the effect of several parameters on the heat transfer from circular cross sectioned helical coiled tubes.

The rates of heat transfer in a straight tube and a helically heat exchanger were compared by Prabhanjan et al. (2002). Authors mentioned that the heat transfer coefficient changes by changing the geometrical configuration. Ko and Ting (2005), used the minimal entropy generation method to evaluate the effect of Reynolds number on the produced irreversibility's in the case of a fully developed laminar flow in a helically tube, the main results is that the entropy generation is insensitive to the coil pitch.

Zheng et al (2000) studied numerically the coupled laminar forced convection and thermal radiation inside a helical pipe. The effects radiative transfer on the flow and heat transfer were

\footnotetext{
* Corresponding Author.

Email Address: lioua enim@yahoo.fr (L. Kolsi)

http://dx.doi.org/10.21833/ijaas.2016.07.004

2313-626X/C 2016 The Authors. Published by IASE.

This is an open access article under the CC BY-NC-ND license

(http://creativecommons.org/licenses/by-nc-nd/4.0/)
}

measured. The comparison shows that thermal radiation has no significant effect on flow and temperature field but it enhances the total heat transfer.

Ko (2006) optimized the mass flow rate for fully developed laminar forced convection of different fluids through circular cross section helically coiled tubes with constant heat flux at walls. The same configuration was treated by Shokouhmand and Salimpour (2007) to evaluate the entropy generation and optimal Reynolds number under constant wall temperature for water and air. An extension of this work was done by the same authors (Shokouhmand and Salimpour, 2007) for more general flow configurations.

Lin and Ebadian (1997) studied numerically the turbulent flow inside a helical coiled tube. They mentioned that helical pitch and curvature affect the streamlines and the heat transfer.

Lin et al. (1997) investigated the laminar forced convection in helical coiled tubes, heat transfer and pressure losses increase as the curvature ratio of the helical coiled tubes increases.

Jayakumar et al. (2008) studied numerically and experimentally the turbulent flow inside helical coils. The authors proposed a correlation of Nusselt number including different parameters.

As extension of their works Jayakumar et al. (2010) studied the effect of the changes of the thermo-physical properties of the fluid. They have shown that the pitch of the helical coil has little effect on Nusselt number 
An experimental investigation was carried out by Seban and McLaughlin (1963). Authors proposed a correlation to determine inner Nusselt number in tube coils for laminar and turbulent flow regimes, based on thermo physical properties of fluid at average temperature.

Patankar et al. (1974) discussed the effect of Dean Number on friction factor and Nusselt number for developing and fully developed flows of helically coiled pipes. Authors mentioned that the centrifugal forces increase the heat transfer.

Rakhsha et al. (2015) investigated experimentally and numerically the turbulent forced convection flow of $\mathrm{CuO}$-water nanofluid. Results indicate that the maximum velocity and $\mathrm{Nu}$ number is located near of the outer wall due to the existence of centrifugal force.

Naphon and Suwagrai (2007) numerically studied the effect of curvature ratios on Nusselt number and flow structure in spirally coiled tubes. Cioncolini and Santini (2006a); (2006b) investigated the transition from laminar to turbulent flow in helically coiled tubes for different curvature ratio.

This work intends to reveal numerically some features that are not obtained in previous researches, by focusing on the heat transfer and flow characteristics inside a helical coiled triangular cross sectioned tube, for different Reynolds numbers, curvatures ratio, and pitch. The flow structure and the distribution of temperature under different flow configurations are provided respectively in the form of streamlines and iso-surfaces.

To validate results, a comparison with results of circular cross sectioned helical tube established by other authors is done. Then a Comparison between the present numerical data with the previous works with circular cross-sectioned helical tubes to evaluate the thermal performance of such configuration.

\section{Geometry, mathematical model and numerical method}

Fig. 1 shows a helical triangular sectioned coiled tube and the unstructured grid. The geometric parameters include the inner triangular cross section side, the curvature radius of the coil $\mathrm{R}$, the coil pitch Pi,

The curvature ratio $\mathrm{d}$ is defined as $\delta=a / R$, and the dimensionless pitch is defined as $P i=b / 2 \pi R$.

The Reynolds number Re, and Nusselt number $\mathrm{Nu}$ are defined as follows:

$$
R e=\rho U_{i} D_{h} / \mu \text { and } N u=h D_{h} / \lambda
$$

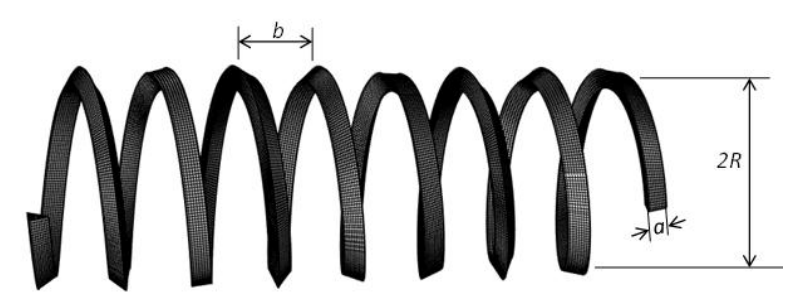

Fig. 1: Geometry and grid of the triangular cross sectioned helical coiled tube
The flow inside the helical coiled tube is considered to be steady and constant thermal properties are assumed. To simulate the turbulent flow and heat transfer, the $\mathrm{k}-\varepsilon$ standard turbulence model is used.

The steady three-dimensional differential equations governing the phenomenon can be written as follows:

Continuity equation:

$\frac{\partial u_{i}}{\partial x_{i}}=0$

Momentum equation:

$\frac{\partial\left(\rho u_{i} u_{j}\right.}{\partial x_{j}}=\frac{\partial}{\partial x_{j}}\left[\left(\mu+\mu_{t}\right)\left(\frac{\partial u_{i}}{\partial x_{j}}+\frac{\partial u_{j}}{\partial x_{i}}\right)-\delta_{i j} \frac{2}{3} \frac{\partial u_{k}}{\partial x_{k}}\right]-$

$\frac{\partial\left(\partial_{i j} P\right.}{\partial x_{i}}$

Energy equation:

$\frac{\partial\left(\rho u_{j} C_{P} T\right)}{\partial x_{j}}=\frac{\partial}{\partial x_{j}}\left[\left(\lambda+\frac{\mu_{t} C_{P}}{P r_{T}}\right) \frac{\partial T}{\partial x_{j}}\right]+\mu \frac{\partial u_{j}}{\partial x_{j}}\left(\frac{\partial u_{i}}{\partial x_{j}}+\frac{\partial u_{j}}{\partial x_{i}}-\right.$

$\left.\delta_{i j} \frac{2}{3} \frac{\partial u_{k}}{\partial x_{k}}\right)+\rho \varepsilon$

Turbulent kinetic energy equation:

$\frac{\partial\left(\rho u_{j} K\right)}{\partial x_{j}}=\frac{\partial}{\partial x_{j}}\left[\left(\mu+\frac{\mu_{t}}{P r_{K}}\right) \frac{\partial K}{\partial x_{j}}\right]+\mu_{t} \frac{\partial u_{j}}{\partial x_{j}}\left(\frac{\partial u_{i}}{\partial x_{j}}+\frac{\partial u_{j}}{\partial x_{i}}\right)-$

$\rho \varepsilon$

Dissipation rate of turbulent kinetic energy:

$\frac{\partial\left(\rho u_{j} \varepsilon\right)}{\partial x_{j}}=\frac{\partial}{\partial x_{j}}\left[\left(\mu+\frac{\mu_{t}}{P r_{\varepsilon}}\right) \frac{\partial \varepsilon}{\partial x_{j}}\right]+\frac{C_{1} \varepsilon \mu_{t}}{K} \frac{\partial u_{i}}{\partial x_{j}}\left(\frac{\partial u_{i}}{\partial x_{j}}+\right.$

$\left.\frac{\partial u_{j}}{\partial x_{i}}\right)-\frac{C_{2} \rho \varepsilon^{2}}{K}$

The turbulent viscosity is defined as:

$\mu_{t}=\frac{C_{\mu} \rho \varepsilon^{2}}{K}$

With:

$C_{\mu}=0.09, C_{1}=1.47, C_{\mu}=1.92, \operatorname{Pr}_{K}=\operatorname{Pr}_{T}=1$,

$P r_{\varepsilon}=1.3$

\subsection{Boundaries conditions}

- $\mathrm{U}=0, \mathrm{v}=0, \mathrm{w}=0, \mathrm{~T}=\mathrm{T}_{\mathrm{w}}$ at all tube walls

- $\mathrm{W}=\mathrm{U}_{\mathrm{i}}, \mathrm{T}=\mathrm{T}_{\mathrm{i}}, \mathrm{K}=\mathrm{K}_{\mathrm{i}, \varepsilon}=\varepsilon_{i}$ at the inlet;

- $\frac{\partial}{\partial n}\left(u_{i}, T, K, \varepsilon\right)=0$ at the outlet.

The bulk temperature, heat transfer rate, local Nusselt number and average Nusselt number are respectively given by:

$$
\begin{aligned}
& T_{b}=\frac{\int_{0}^{A} w T d A}{\int_{0}^{A} w d A}, q_{w}=\lambda\left(\frac{\partial T}{\partial n}\right), \\
& N u=\frac{D_{h} q_{w}}{\lambda\left(T_{w}-T_{b}\right)} \text { and } N u_{a v}=\frac{1}{2 \pi} \int_{0}^{2 \pi} N u d \emptyset
\end{aligned}
$$

To represent results, the dimensionless temperature and velocity are defied as:

$\Theta=\frac{T-T_{w}}{T_{b}-T_{w}}, V=\frac{w}{U_{i}}$

\section{Results and discussions}

The effect of different parameters on the turbulent flow forced convective heat transfer and flow developments characteristics of water inside a helical triangular sectioned coiled tube is demonstrated. Results of the numerical simulations are presented, in term of Nusselt number, streamlines, velocity field and temperature field. 
A validation of the used software was done by comparing the results of Nusselt number for the case of helical circular cross sectioned coiled tube with other works presented in literature.

In fact Fig. 2 shows a comparison between our results and those presented by Jayakumar et al. (2008) and Rogers and Mayhew (1964). Results indicate an acceptable concordance especially for high Reynolds numbers.

Effect of Re number for fixed Pitch (Pi=0.318) and curvature ratio $(\delta=0.115)$ on the fully developed dimensionless velocity and temperature profiles is demonstrated in Figs. 3 and 4. It could be concluded that the increase of Re number affect the structure of dimensionless velocity and temperature by moving the maximum velocity point toward the outer wall corner and makes the outer wall gradients more severe. However, the geometry of the curved pipe (d) changes the general pattern of temperature and velocity contours.

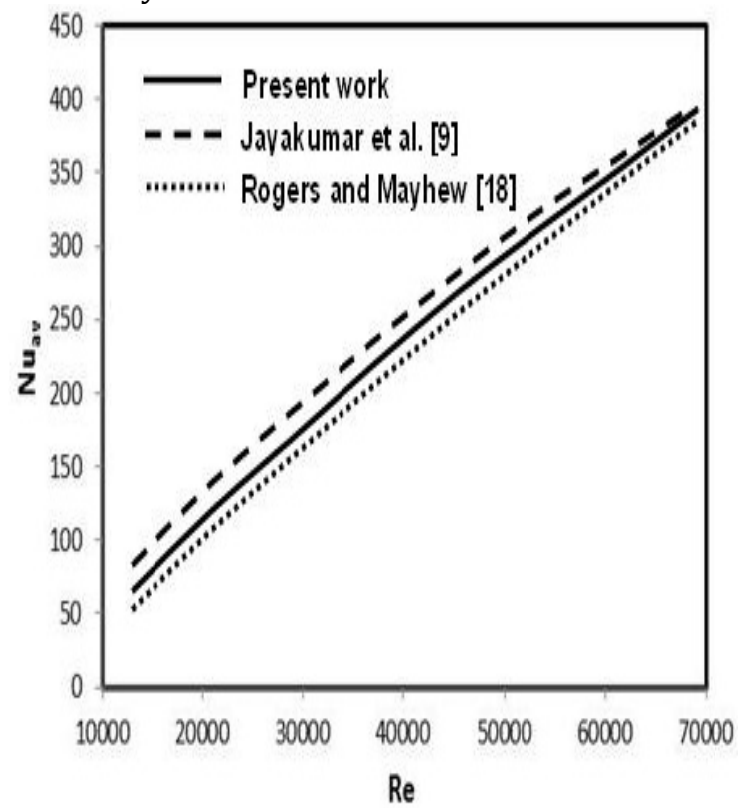

Fig. 2: Comparison of obtained Nuav with results of Jayakumar et al. (2008) and Rogers and Mayhew (1964)
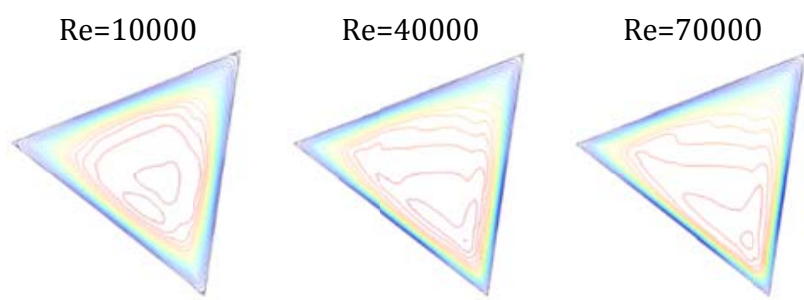

Fig. 3: Fully developed dimensionless velocity profiles for different Re
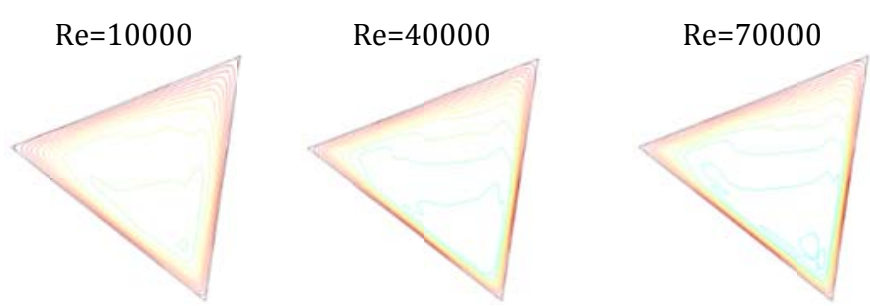

Fig. 4: Fully developed dimensionless temperature profiles for different $\mathrm{Re}$
Effect of the curvature ratio on the fully developed velocity and temperature profiles at fixed Reynolds number $(\mathrm{Re}=40000)$ and fixed Pitch number $\mathrm{Pi}=0.636$, was demonstrated in the left part of Figs. 4 and 5 respectively. It's well known that the increase of curvature ratio generates higher centrifugal forces which cause the displacement of dimensionless velocity and dimensionless temperature closer to the outer wall. This displacement is more pronounced for $\delta>1 / 10$. It's also noticed that as the increase of the curvature ratio provokes an intensification of gradient of temperature and velocity near of the walls. The Right part of these figures presents the different stage of the developing dimensionless velocity and dimensionless temperature profiles. Regarding the effect of the pitch on these profiles, it is found that there is no sensitive modification of the structure by changing the Pitch number as shown in Fig. 6, which presents the velocity profile for different pitch numbers. In fact the maximum of the velocity is almost near of the center of the cross section especially for higher pitch numbers.

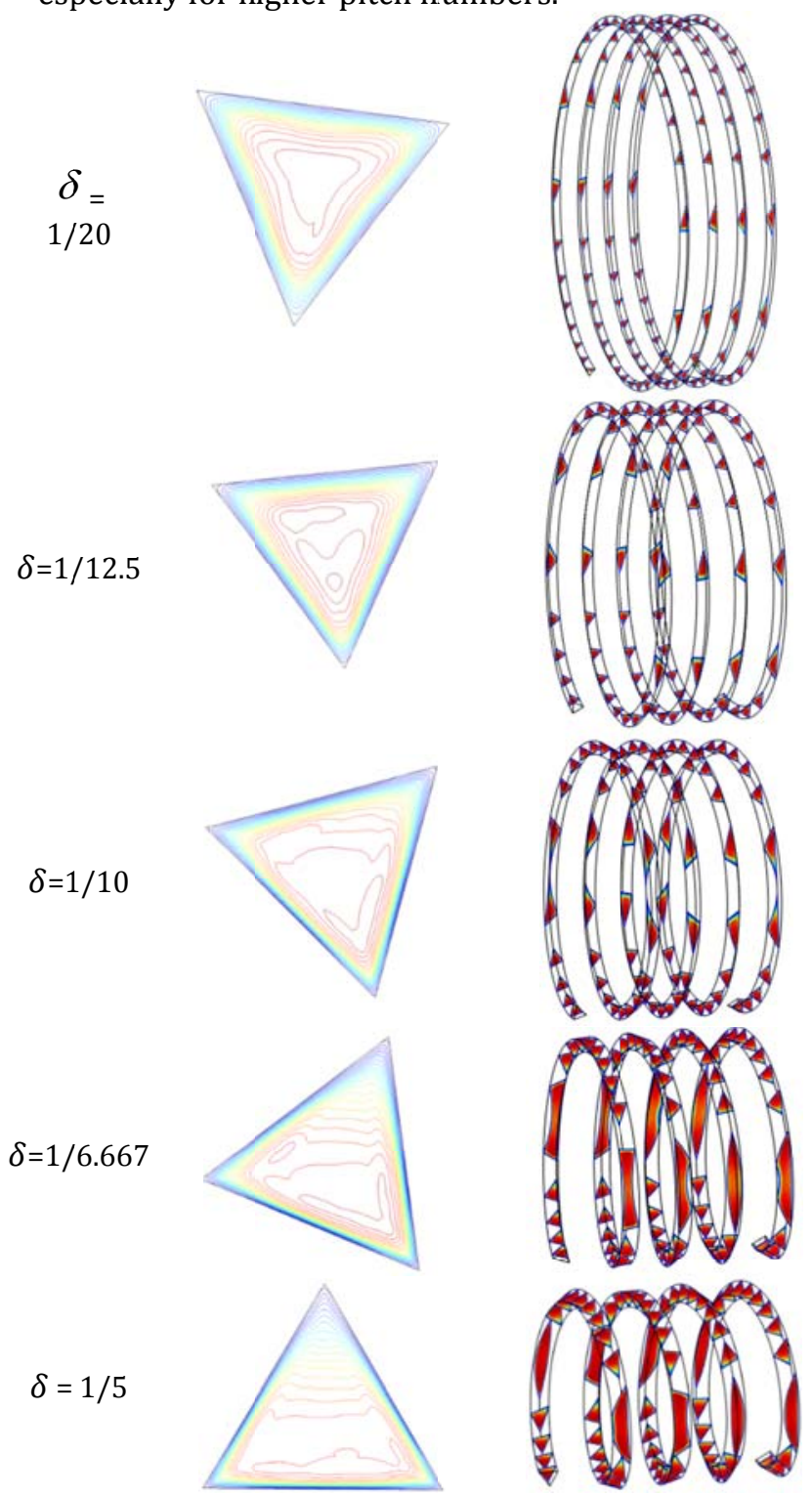

Fig. 5: Fully developed velocity profiles for $\mathrm{Re}=40000, \mathrm{Pi}$ $=0.318$ and different curvature ratio 


$$
\delta=1 / 20
$$

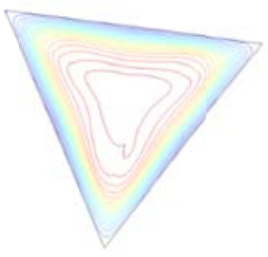

$\delta=$

$1 / 12.5$

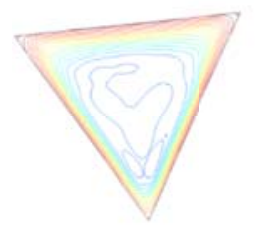

$\delta=1 / 10$
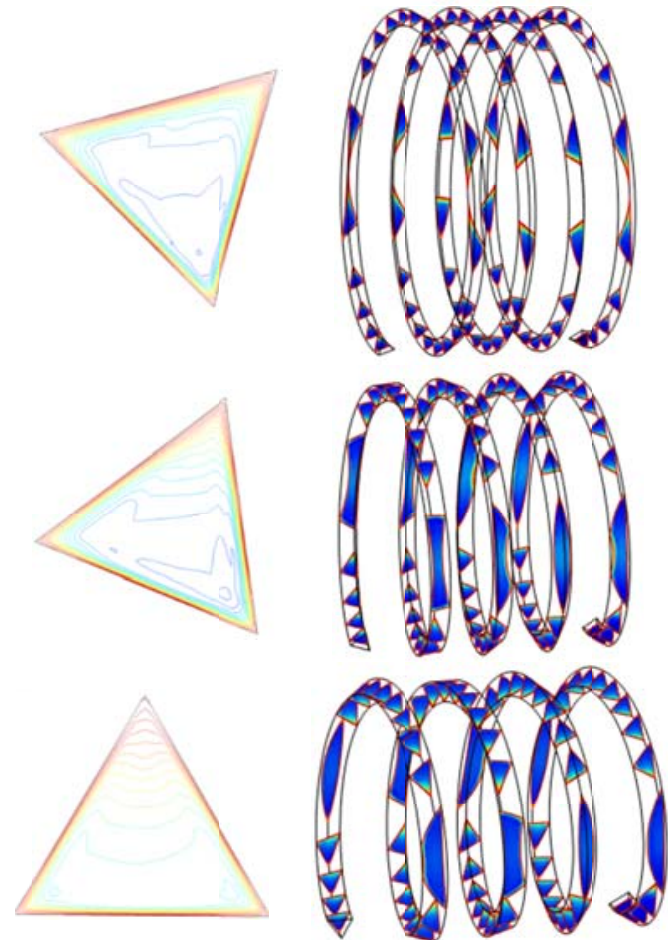

Fig. 6: Fully developed temperature profiles for $\mathrm{Re}=$ $40000, \mathrm{Pi}=0.636$ and different curvature ratio

For illustration and to show the 3D aspect of the flow, Fig. 7 presents some particles trajectories for $\operatorname{Re}=40000, \delta=0.115$ and $\mathrm{Pi}=0.636$, it's clear that the flow follows the spiral geometry of the coiled tube.

Fig. 8 presents a comparison between the average Nusselt number for triangular and circular cross sections. This figure shows that the heat transfer using a circular cross section is more important. This result is expected given that it is the case for linear tubes.
$\mathrm{Pi}=$

0.159
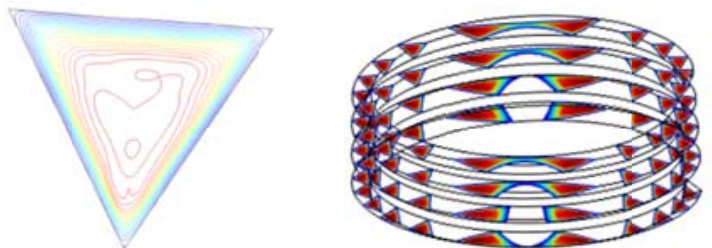

$\mathrm{Pi}=$

0.636
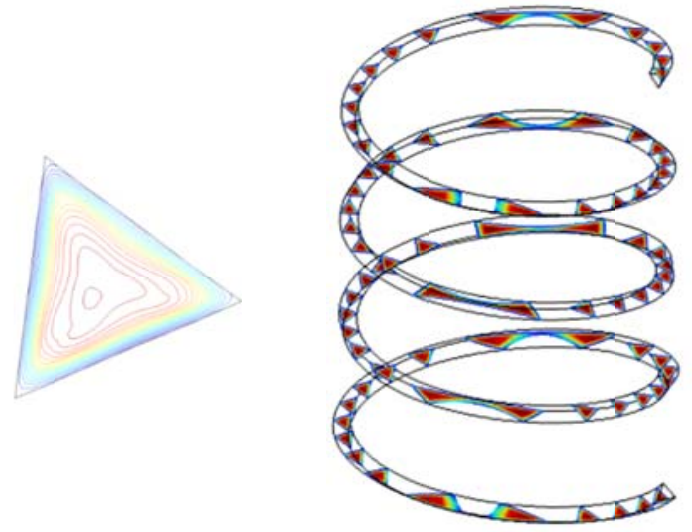

$\mathrm{Pi}=$

1.273
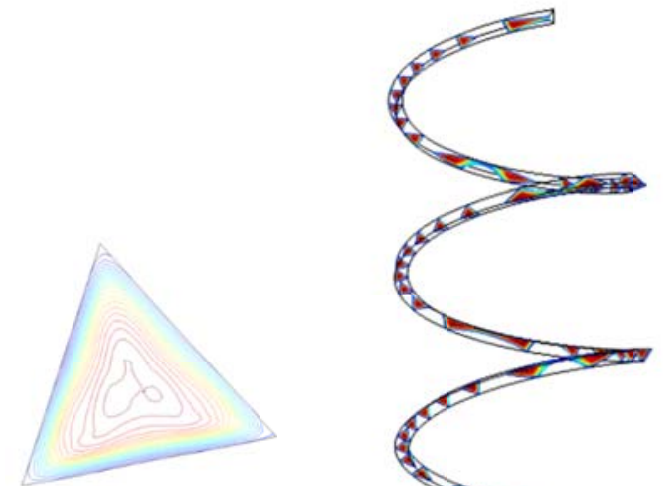

$\mathrm{Pi}=$

2.547
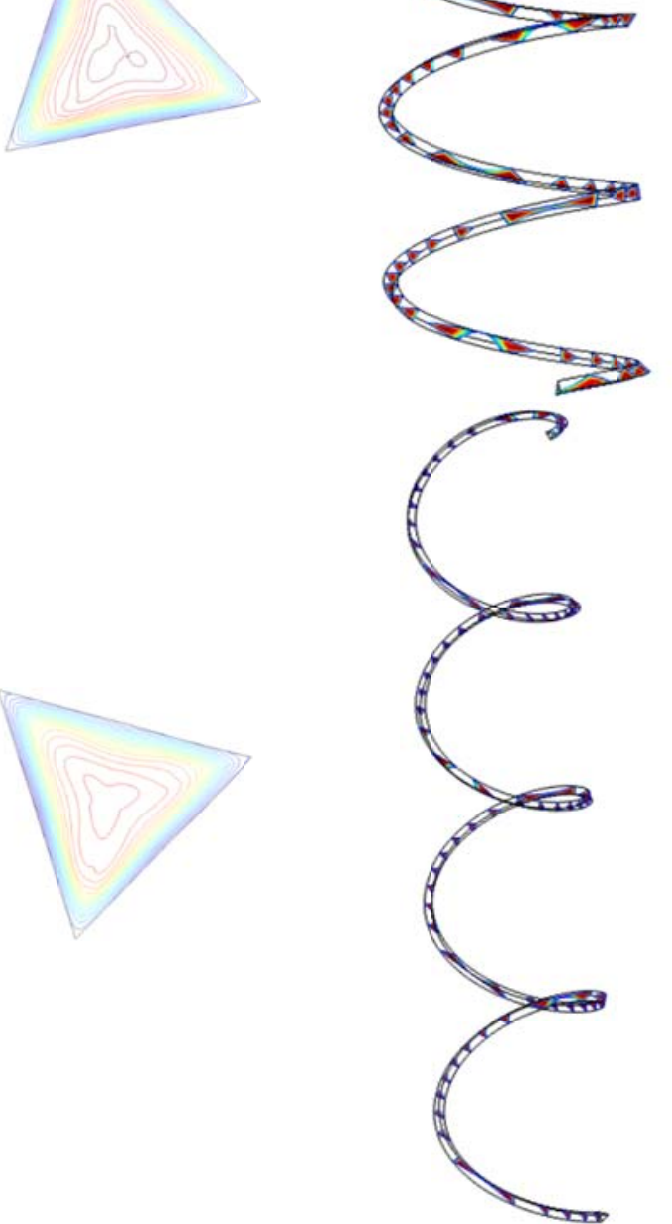

Fig. 7: Fully developed velocity" profiles for $\operatorname{Re}=40000, \delta=$ 0.115 and different Pitch number 


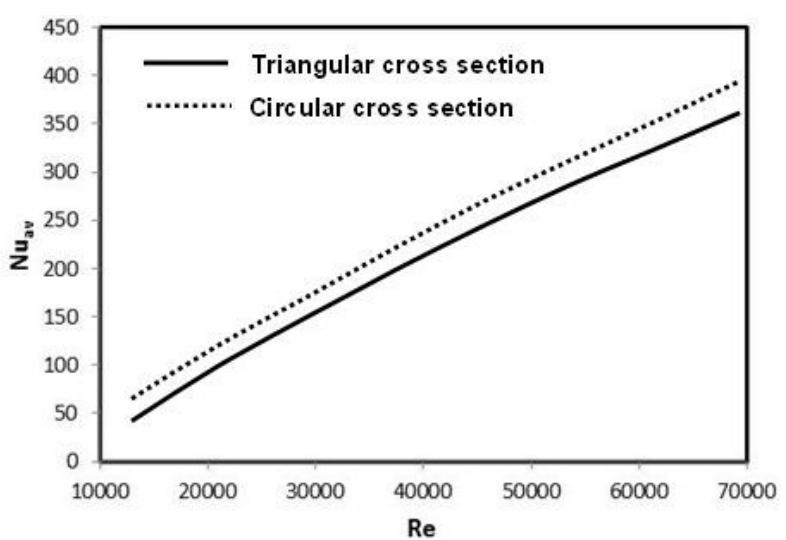

Fig. 8: Comparison of results of average Nusselt number for triangular and circular cross sections

\section{Conclusion}

Three-dimensional turbulent flow and heat transfer in a helical triangular cross sectioned coiled tube have been investigated in this paper. The numerical computations show the developments and distributions of heat transfer and flow fields in the tube. These profiles are altered by the effect of curvature inducing the centrifugal forces.

The effects of various flow and geometric parameters on flow, temperature field and heat transfer were studied and. The most important results can be summarized as

- The increase of Re number affect the structure of dimensionless velocity and temperature by moving the maximum velocity point toward the outer wall corner and makes the outer wall gradients more severe..

- The increase of curvature ratio generates higher centrifugal forces which cause the displacement of dimensionless temperature and dimensionless velocity closer to the outer wall.

- The increase of the curvature ratio provokes an intensification of gradient of temperature and velocity near of the walls.

- There is no sensitive modification of the structure by changing the Pitch number

- The heat transfer using an ordinary circular cross section is found to be more important.

\section{Acknowledgment}

The authors would like to thank the Deanship of the Scientific Research of University of Hail, Saudi Arabia for funding and supporting this research project (E15-ME 2014).

\section{Nomenclature}

$\begin{array}{cc}\mathrm{a} & \text { inner triangular cross section side, } \mathrm{m} \\ \mathrm{A} & \text { cross-section area, } \mathrm{m} \\ \mathrm{b} & \text { coil pitch, } \mathrm{m} \\ \mathrm{C}_{1}, \mathrm{C}_{2}, & \text { turbulent model constant } \\ \mathrm{C}_{3} & \text { specific heat capacity, } \mathrm{J} /(\mathrm{kg} \mathrm{K}) \\ \mathrm{Cp} & \text { Hydraulic diameter } \\ \mathrm{D}_{\mathrm{h}} & \text { thermal conductivity of fluid, } \mathrm{W} /(\mathrm{m} \mathrm{K}) \\ \lambda & \end{array}$

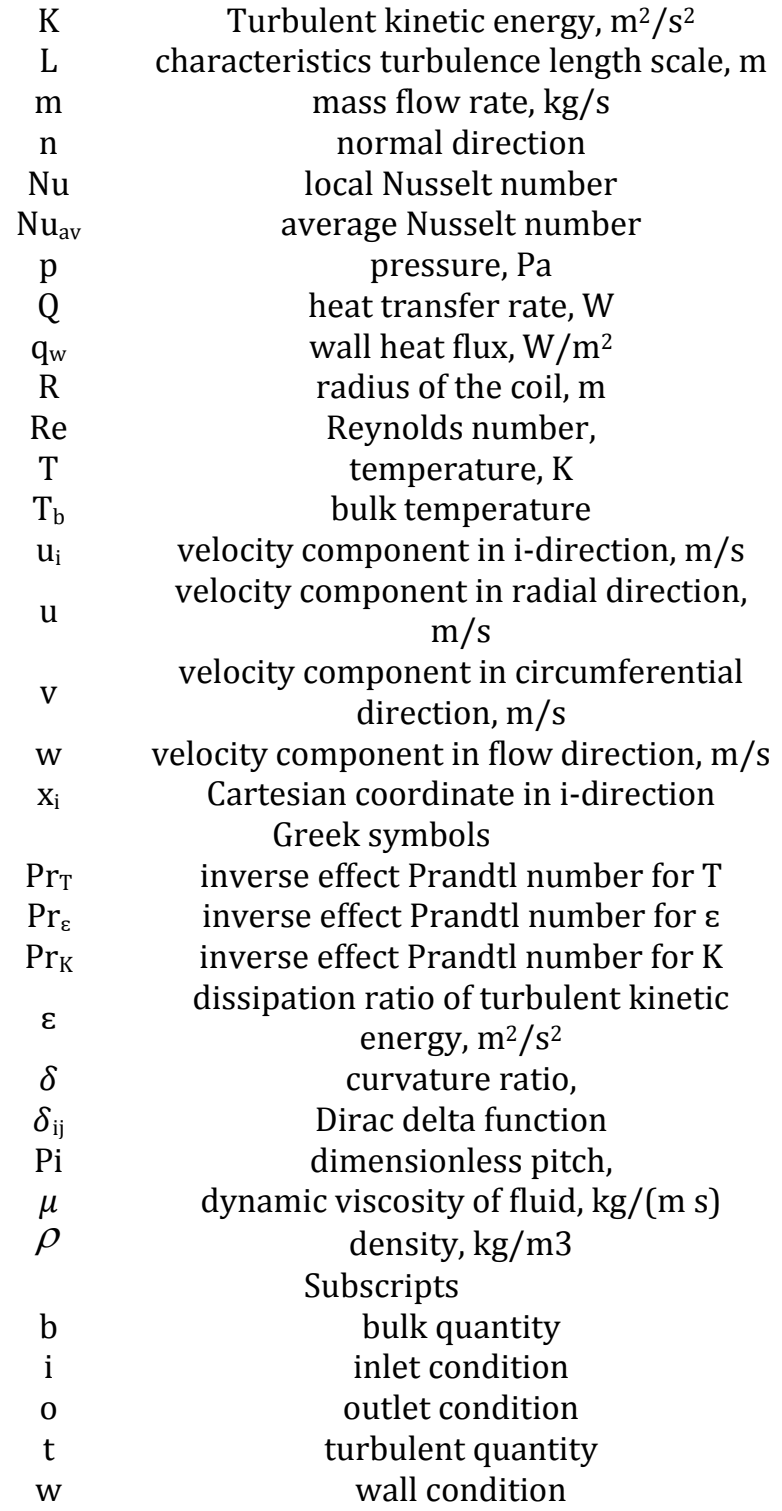

\section{References}

Cioncolini A and Santini L (2006). An experimental investigation regarding the laminar to turbulent flow transition in helically coiled pipes. Experimental Thermal and Fluid Science, 30(4): 367-380.

Cioncolini A and Santini L (2006). On the laminar to turbulent flow transition in diabatic helically coiled pipe flow. Experimental Thermal and Fluid Science, 30(7): 653-661.

Jayakumar JS, Mahajani SM, Mandal JC, Iyer KN and Vijayan PK (2010). CFD analysis of single-phase flows inside helically coiled tubes. Computers and Chemical Engineering, 34(4): 430-446.

Jayakumar JS, Mahajani SM, Mandal JC, Vijayan PK and Bhoi R (2008). Experimental and CFD estimation of heat transfer in helically coiled heat exchangers. Chemical Engineering Research and Design, 86(3), 221-232.

Ko TH (2006). Thermodynamic analysis of optimal mass flow rate for fully developed laminar forced 
convection in a helical coiled tube based on minimal entropy generation principle. Energy Conversion and Management, 47(18): 3094-3104.

Ko TH and Ting K (2005). Entropy generation and thermodynamic optimization of fully developed laminar convection in a helical coil. International Communications in Heat and Mass Transfer, 32(1): 214-223.

Lin CX and Ebadian MA (1997). Developing turbulent convective heat transfer in helical pipes. International Journal of Heat and Mass Transfer, 40(16), 3861-3873.

Lin CX, Zhang P and Ebadian MA (1997). Laminar forced convection in the entrance region of helical pipes. International Journal of Heat and Mass Transfer, 40(14): 3293-3304.

Naphon P and Suwagrai J (2007). Effect of curvature ratios on the heat transfer and flow developments in the horizontal spirally coiled tubes. International Journal of Heat and Mass Transfer, 50(3): 444-451.

Patankar SV, Pratap VS and Spalding DB (1974). Prediction of laminar flow and heat transfer in helically coiled pipes. Journal of Fluid Mechanics, 62(03): 539-551.

Prabhanjan DG, Raghavan GSV and Rennie TJ (2002). Comparison of heat transfer rates between a straight tube heat exchanger and a helically coiled heat exchanger. International Communications in Heat and Mass Transfer, 29(2): 185-191.

Rakhsha M, Akbaridoust F, Abbassi A and Majid SA (2015). Experimental and numerical investigations of turbulent forced convection flow of nano-fluid in helical coiled tubes at constant surface temperature. Powder Technology, 283: 178-189.

Rogers GFC and Mayhew YR (1964). Heat transfer and pressure loss in helically coiled tubes with turbulent flow. International Journal of Heat and Mass Transfer, 7(11): 1207-1216.

Seban RA and McLaughlin EF (1963). Heat transfer in tube coils with laminar and turbulent flow. International Journal of Heat and Mass Transfer, 6(5): 387-395.

Shokouhmand H and Salimpour MR (2007). Entropy generation analysis of fully developed laminar forced convection in a helical tube with uniform wall temperature. Heat and Mass Transfer, 44(2): 213-220.

Zheng B, Lin CX and Ebadian MA (2000). Combined laminar forced convection and thermal radiation in a helical pipe. International Journal of Heat and Mass Transfer, 43(7): 1067-1078. 